\title{
Optimal detection of solitons with timing jitter
}

\author{
Keang-Po Ho \\ Institute of Communications Engineering and Department of Electrical Engineering, National Taiwan University, \\ Taipei 106, Taiwan
}

Received November 29, 2004; revised manuscript received April 14, 2005; accepted April 23, 2005

\begin{abstract}
When a soliton signal is detected by the maximum-likelihood principle, other than walk-out of the bit interval, timing jitter does not degrade the performance of the receiver. When the maximum-likelihood detector (MLD) is simulated by using the importance sampling method, even with a timing-jitter standard deviation the same as the full width at half-maximum of the soliton, the signal-to-noise (SNR) penalty is just about $0.2 \mathrm{~dB}$. The MLD performs far better than the conventional scheme to lengthen the decision window with SNR degradation proportional to the increase of the window width. (C) 2005 Optical Society of America
\end{abstract}

OCIS codes: $060.5530,190.5530,060.4370$.

\section{INTRODUCTION}

The Gordon-Haus timing jitter ${ }^{1}$ limits the transmission distance of a soliton communication system. Without any in-line control, the arrival time of the soliton has a variance increasing cubically with the transmission distance. Although timing jitter can be reduced using an in-line filter, ${ }^{2,3}$ an in-line modulator, ${ }^{4}$ or other methods, ${ }^{5}$ it remains one of the major limitations for soliton transmission systems. For a system with in-line timing-jitter control, the transmission distance can be extended to long distances. ${ }^{4,6}$ When amplifier noise is accumulated with the transmission distance, the signal-to-noise ratio (SNR) reduces for the extended distance, and the optical amplifier noise becomes the primary limitation of the system.

Instead of another method among many methods ${ }^{5}$ to reduce timing jitter in the fiber link, this paper discusses a method to detect the soliton signal with amplifier noise and timing jitter at the receiver. To reduce the sensitivity penalty due to timing jitter, we apply the optimal detector regardless if timing jitter is controlled in the fiber link. The optimal method to detect the presence or absence of a soliton with timing jitter is derived, to our knowledge, for the first time.

Previously, the decision window of the soliton was widened significantly to reduce the impact of timing jitter. ${ }^{1,5}$ However, the widening of the decision window degrades the equivalent SNR of the decision variable before the decision circuits. For example, if the decision window is doubled to twice wider than necessary, the SNR is halved, giving a $3 \mathrm{~dB}$ SNR penalty to the system and requiring a twice-larger received signal for the same performance. With an electroabsorption modulator as an optical timedomain demultiplexer to provide a widened decision window, ${ }^{7}$ the timing window may reach $80 \%$ of the bit interval for timing-jitter resilient reception. ${ }^{8}$

A decision window is equivalent to an integator for the optical intensity with an integration interval the same as the decision window. An electrical low-pass filter is equivalent to a weighted decision window with the weight equal to the impulse response of the electrical filter. Even for a soliton without timing jitter, a rectangular or weighted decision window is inferior to the matchedfilter-based receiver that maximizes the SNR. A method to combat timing jitter without leading to significant increase in the SNR penalty is investigated here on the basis of maximum-likelihood detection (MLD).

When the MLD is derived from the first principle, an optical matched filter is used before the photodetector, followed by nonlinear signal processing. In conventional detection theory, ${ }^{9}$ the matched-filter-based receiver maximizes the output SNR. Optical matched filters can be implemented optically with an impulse response identical to the pulse shape. ${ }^{10,11}$ The signal processing after the photodetector is derived on the basis of the MLD decision rule to minimize the error probability.

The remaining parts of this paper are organized as follows. Section 2 derives the MLD for a soliton with timing jitter and shows that it can be implemented using some optimal optical and electrical filters, together with nonlinear signal processing. Section 3 shows the performance of the MLD for a soliton based on numerical simulation. Section 4 is the conclusion of the paper.

\section{MAXIMUM-LIKELIHOOD DETECTION OF SOLITONS}

The optimal detection of a signal should be based on the maximum-likelihood criterion to minimize the probability of decision error. For a soliton signal, the MLD should minimize the error probability on the detection of the presence or absence of a soliton pulse. If the digits of 1 and 0 are represented by the presence or absence of a soliton and it is assumed that 1 or 0 is transmitted with equal probability, MLD decides the presence of a soliton by $p[r(t) \mid 1]>p[r(t) \mid 0]$, where $r(t)$ is the received signal and $p[r(t) \mid 1]$ and $p[r(t) \mid 0]$ are the probability of having a received signal of $r(t)$ given the condition with the presence or absence of a soliton, respectively. ${ }^{9}$ Similarly, the absence of a soliton is decided if $p[r(t) \mid 1]<p[r(t) \mid 0]$.

In a soliton communication system, the received signal can be represented as 


$$
r(t)=a_{k} s\left(t-t_{0}\right) \exp (j \phi)+n(t)
$$

where $a_{k} \in\{0,1\}$ for the absence or presence of the soliton, $s(t)=\operatorname{sech}(1.76 t)$ is the normalized soliton pulse with unity FWHM pulse width, $t_{0}$ is a random variable representing the timing jitter, $\phi$ is the random phase due to the propagation delay and soliton phase jitter, and $n(t)$ is the additive complex-value white Gaussian noise. The noise of $n(t)$ is induced by optical amplifiers, and $t_{0}$ is GordonHaus timing jitter with and without in-line control. Only the noise with the same polarization as the soliton is considered here by assuming a polarized receiver. The noise from orthogonal polarization can be included with small modification. For a phase-insensitive receiver, the phase of $\phi$ is assumed to be uniformly distributed from 0 to $2 \pi$.

The expression of Eq. (1) clearly defines the problem addressed in this paper. The timing jitter of $t_{0}$ by itself and the noise of $n(t)$ are not reduced by the receiver. The receiver just minimizes the impact of both $t_{0}$ and $n(t)$, provided that both of them appear at the receiver input together with the soliton of $s(t)$. The fiber link, or the medium between transmitter and receiver, may or may not use in-line time-jitter control.

The signal of $s(t)$ in Eq. (1) is not necessary as a soliton as long as its pulse shape is well defined. The timing jitter of $t_{0}$ is also not necessarily Gordon-Haus timing jitter, but its probability density function must be known. Regardless of the sources of time jitter, we reduce its impact on the system. For example, pulse-to-pulse collision in a dispersive system also gives timing jitter. ${ }^{12,13}$ However, the probability density of the timing jitter is not known or must be found using extensive calculations. ${ }^{14}$ Only a soliton with the Gordon-Haus timing jitter is discussed here because of the availability of signal and timing-jitter models.

Usually, soliton propagation with noise is studied by the first-order perturbation theory of the soliton ${ }^{5,15-17}$ in which amplifier noise is distributively projected to amplitude and frequency jitter along the fiber. When the firstorder soliton perturbation is linearized, ${ }^{5}$ there is no difference between whether amplitude jitter is a distributed contribution along the fiber link or a lumped contribution at the beginning or the end of the fiber. An example is $n(t)=n_{1}(t)+n_{2}(t)$, with $n_{1}(t)$ and $n_{2}(t)$ from the first half and second half of the fiber link, respectively. The linearized model of soliton perturbation uses only the combined noise of $n(t)$ and the order of $n_{1}(t)$ and $n_{2}(t)$ does not affect the results; for example, the results do not change if $n_{1}(t)$ is first applied and then $n_{2}(t)$ or if $n_{2}(t)$ is first applied and then $n_{1}(t)$. Of course, if first-order large-signal perturbation is used, there is a small difference between the distributed or lumped model. ${ }^{5,18,19}$ The received signal of Eq. (1) assumes all amplifier noise at the end of the fiber link. The timing jitter of $t_{0}$ and phase jitter are independent of the amplitude jitter. ${ }^{5}$ If phase jitter is also included in $\phi$, the model of Eq. (1) is not different from the linearized model for first-order soliton perturbation.

As shown in Ref. 9, Section 6.4, given a phase of $\phi$ and $a_{k}=1$ with the presence of a soliton, the probability density of the received signal is $p\left[r(t) \mid 1, t_{0}, \phi\right]=\alpha \exp \left[-\frac{1}{N_{0}} \int_{-\infty}^{\infty}\left|r(t)-s\left(t-t_{0}\right) \exp (j \phi)\right|^{2} \mathrm{~d} t\right]$,

where $\alpha$ is a proportional constant and $N_{0} / 2$ is the spectral density of $n(t)$. The above probability density of Eq. (2) makes no assumption about the receiver but just assumes a white Gaussian noise of $n(t)$.

If the soliton is detected by a photodetector, the phase of $\phi$ in Eq. (1) does not affect the system performance. With a detail provided in Ref. 9, Section 7.2, after averaging over the random phase of $\phi$, one finds that the probability density of the received signal is equal to

$p\left[r(t) \mid 1, t_{0}\right]=\alpha \exp \left[-\frac{1}{N_{0}} \int_{-\infty}^{\infty}|r(t)|^{2} \mathrm{~d} t-\frac{E}{N_{0}}\right] I_{0}\left[\frac{2 \sqrt{E} q\left(t_{0}\right)}{N_{0}}\right]$,

where $I_{0}()$ is the zero-order modified Bessel function of the first kind, $E=\int_{-\infty}^{\infty} s^{2}(t) \mathrm{d} t$ is the energy per soliton pulse, and $q\left(t_{0}\right)$ is equal to

$$
q\left(t_{0}\right)=\left|\int_{-\infty}^{\infty} r(t) s^{*}\left(t-t_{0}\right) \mathrm{d} t\right|, \quad q\left(t_{0}\right) \geqslant 0 .
$$

The calculation of $q\left(t_{0}\right)$ is based on the integration of $\int_{-\infty}^{\infty} r(t) s\left(t-t_{0}\right) \mathrm{d} t$ that is the same as the output of an optical matched filter. Here, the matched filter output cannot be used directly for detection purposes, but the optical matched filter is just a step for the MLD. Nonlinear signal processing is required, for example, for the absolute value to get a positive $q\left(t_{0}\right)$.

Similarly, when $a_{k}=0$ without a soliton, we obtain

$$
p[r(t) \mid 0]=\alpha \exp \left[-\frac{1}{N_{0}} \int_{-\infty}^{\infty}|r(t)|^{2} \mathrm{~d} t\right] .
$$

The probability density of Eq. (3) is similar to the Rice distribution, and that of Eq. (5) is similar to the Rayleigh distribution. These two distributions are well known in the field of signal detection. ${ }^{9}$

If the probability density of timing jitter is $p_{T}\left(t_{0}\right)$, we obtain

$$
p[r(t) \mid 1]=\int_{-\infty}^{\infty} p\left[r(t) \mid 1, t_{0}\right] p_{T}\left(t_{0}\right) \mathrm{d} t_{0} .
$$

The maximum-likelihood criterion gives the decision rule of

$$
p[r(t) \mid 1] \underset{0}{\gtrless} p[r(t) \mid 0]
$$

for the presence or absence of a soliton with time jitter. After some algebra, the decision rule becomes

$$
\int_{-\infty}^{\infty} I_{0}\left[\frac{2 \sqrt{E} q\left(t_{0}\right)}{N_{0}}\right] p_{T}\left(t_{0}\right) \mathrm{d} t_{0} \underset{0}{\gtrless} \exp \left(\frac{E}{N_{0}}\right) .
$$

In the decision rule of expression (8), the integration of $|r(t)|^{2}$ in Eqs. (3) and (5) and the constant of $\alpha$ cancel each other. 


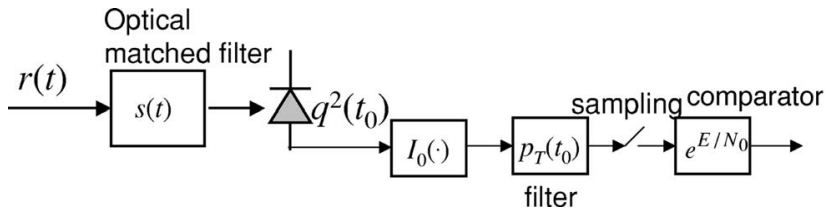

Fig. 1. Schematic of the MLD of the presence or absence of a soliton with timing jitter. This schematic diagram shows the implementation of the decision rule of expression (8). Comp, $\mathbf{x x x}$.

The decision rule of expression (8) together with the parameter $q\left(t_{0}\right)$ calculated by Eq. (4) can be implemented by the schematic block diagram of Fig. 1 . The received signal first passes through an optical matched filter having an impulse response equal to the soliton pulse of $s(t)$ [or $s($ $-t$ ) for an asymmetric pulse]. The output of the optical matched filter is $q\left(t_{0}\right) \exp (j \phi)$. The output of the optical matched filter converts into an electrical signal with a photodetector. The photodetector gives an output proportional to the square of $q\left(t_{0}\right)^{2}$. The implementation of the correlation of Eq. (4) by using a matched filter can be found, for example, in Ref. 9, Chap. 6. Notice that $q\left(t_{0}\right)^{2}$ can give $q\left(t_{0}\right)$ without loss of any information. With the output of $q\left(t_{0}\right)^{2}$ from the photodetector, the value of $I_{0}\left[2 \sqrt{E} q\left(t_{0}\right) / N_{0}\right]$ in expression (8) can be found. The integration in expression (8) with respect to $t_{0}$ is again implemented using an electrical filter with an impulse response of $p_{T}\left(t_{0}\right)$, whose output is sampled at the right time. In Fig. 1, the probability density of $p_{T}\left(t_{0}\right)$ does not need to be Gaussian distributed, ${ }^{18,20}$ but it must be symmetrical with respect to zero. After the sampler, the presence or absence of the soliton is decided when compared with the scalar value of $\exp \left(E / N_{0}\right)$. The filter with impulse response of $p_{T}\left(t_{0}\right)$ may be called an electrical matched filter with an impulse response matched to the probability density of the timing jitter. The nonlinear operation of the MLD includes the square operation of the photodetector with respect to the electric field and the calculation of $I_{0}\left[2 \sqrt{E} q\left(t_{0}\right) / N_{0}\right]$.

The block diagram of Fig. 1 is an implementation of the optimal decision rule of expression (8). The decision rule of expression (8) indicates that an optical matched filter should be used together with the processing shown in Fig. 1. In practice, an optical matched filter was used for optimal performance in Refs. 10,11 for the detection of a signal without timing jitter. The signal processing after the photodetector can be implemented, for example, by digital signal processing.

Practical optical and electrical components of Fig. 1 may have small differences with the decision rule of expression (8) owing to implementation errors. Although we cannot list and analyze all those differences in this paper, the optimal decision rule of expression (8) is compared with the conventional implementation of the widening of the decision window.

\section{PERFORMANCE EVALUATION}

Without timing jitter or $p_{T}\left(t_{0}\right)=\delta\left(t_{0}\right)$, the right-hand side of expression (8) becomes $\left.I_{0}\left(2 \sqrt{E} q / N_{0}\right)\right|_{t_{0}=0}$, and the decision rule of expression (8) can be simplified to a quadratic detector (Ref. 9, Section 8.3). The quadratic detector is

$$
q^{2} \underset{0}{\gtrless} q_{\text {th }}^{2},
$$

with $q_{\text {th }}$ as the optimal threshold without timing jitter. With a performance the same as that for noncoherent detection of an amplitude-shift keying signal, the error probability can be calculated using the well-known Marcum $Q$ function. ${ }^{21,22}$ The error probability for the case without timing jitter is shown in Fig. 2 as a dashed line. The error probability of Fig. 2 is shown as a function of the SNR, given by the ratio of $E / N_{0}$. The threshold of detection is calculated using expression (8) with $p_{T}\left(t_{0}\right)$ $=\delta\left(t_{0}\right)$. An error probability of $10^{-9}$ requires an SNR of about $18.9 \mathrm{~dB}$ for a soliton signal.

The performance of the MLD of expression (8) does not lead to a simple analytical error probability for a soliton with timing jitter. Numerical simulation is conducted when the timing jitter is zero-mean Gaussian distributed with variance of $\sigma_{t}^{2}$. The performance of the system is determined by the variance of $\sigma_{t}^{2}$ and the SNR, maybe together with some parameters of the signal. Both the SNR of $E / N_{0}$ and the variance of the timing jitter of $\sigma_{t}^{2}$ are functions of the fiber link configuration. Although the calculation of the SNR is a standard procedure for system design, the variance of $\sigma_{t}^{2}$ further depends on the method of timing-jitter control.

The simulated error probabilities are shown in Fig. 2 with $\sigma_{t}$ normalized to the FWHM of the soliton. Figure 2 shows that the MLD for a soliton with timing jitter has a small SNR penalty for $\sigma_{t}$ comparable with the FWHM of the soliton.

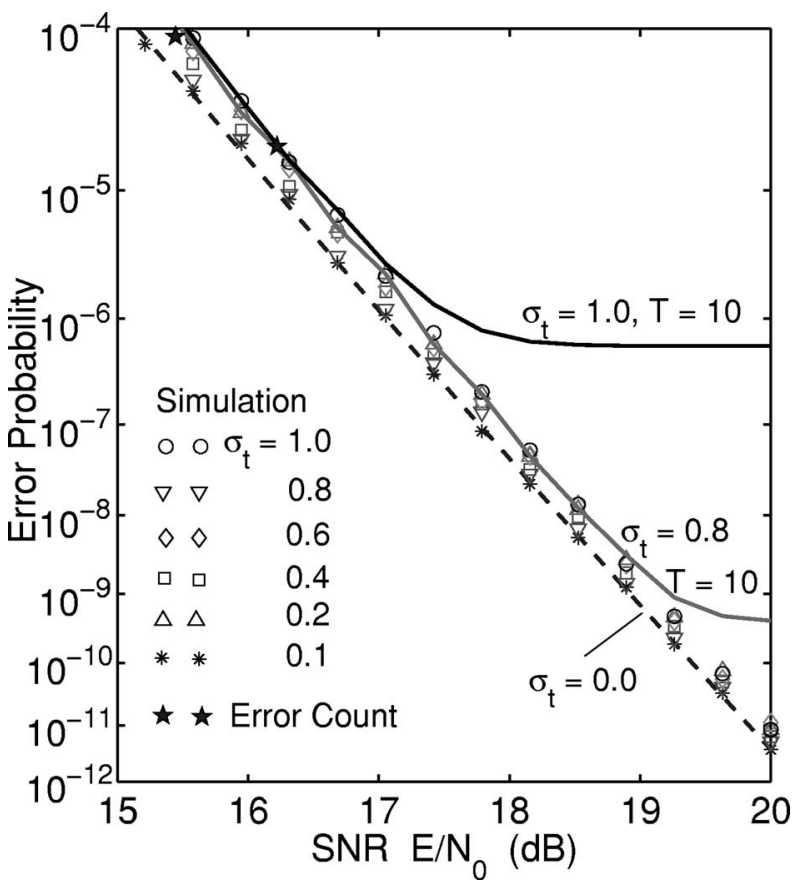

Fig. 2. Simulated error probability of the MLD for a soliton with timing jitter. Various markers (except the two five-point stars) are the error probability from an importance sampling simulation. The two five-point stars are from a Monte Carlo error count for $\sigma_{t}=1$. The dashed line is the theoretical error probability without timing jitter. Solid curves include the walk-out probability that the timing jitter is outside the bit interval. 
To investigate those cases with small error probabilities around $10^{-9}$, a numerical simulation cannot be conducted directly using the method of Monte Carlo error count. The simulation of Fig. 2 uses importance sampling, similar to the methods of Refs. 19, 23, and 24. The received signal is a soliton with different timing jitter according to the Gaussian distribution with variance of $\sigma_{t}^{2}$. The noise sample after the optical matched filter of Fig. 1 with a time corresponding to the peak optical intensity is generated on the basis of uniform distribution. Other noise samples are generated by Gaussian distribution with a correlation depending on the optical matched filter. Each error count is weighted according to the probability difference between the actual Gaussian noise samples and the generated noise samples. ${ }^{19,23}$ Other than adding a biased noise sample after the optical matched filter of Fig. 1 instead of the actual signal with amplifier noises before the filter, the numerical simulation followed closely the decision rule of expression (8). The sampling time before the comparator is chosen by the optimal time to maximize the signal power for the case without both noise and timing jitter.

The error probability calculated from the simulation is shown in Fig. 2 using different markers for a timing-jitter standard deviation of $\sigma_{t}$ from 0.1 to 1.0 of the FWHM of the soliton. Even with a soliton having a large timing jitter of $\sigma_{t}=1.0$, the SNR penalty is just about $0.2 \mathrm{~dB}$ compared with the case without timing jitter (dashed line).

Figure 2 also shows the error probability, taking into account the probability that the soliton may have a timing jitter outside the bit interval when $\sigma_{t}=0.8,1.0$ and the bit interval is ten times the FWHM of the soliton. The bit interval of $T=10$ is chosen for convenience. ${ }^{1}$ For $\sigma_{t}<0.8$, the walk-out probability does not affect the overall error probability and is not shown in Fig. 2. From Fig. 2, a soliton with a large timing jitter is mainly affected by the walk-out probability, especially for a system with a bit interval just four to six times the FWHM of the soliton. Unlike the receiver with widening decision window, the simulation results of Fig. 2 show that the receiver schematic of Fig. 1 does not give a large SNR penalty.

The importance sampling method biases the noise sampling after the optical matched filter to speed up the simulation. With noise samples independent of one another, the biasing of only one noise sample before the optical matched filter does not speed up the simulation. The biasing of all noise samples before the optical filter speeds up the simulation, but the evaluation of weighting is not as simple as the biasing of only one noise sample. In addition to importance sampling, simulation is also conducted the raw method of Monte Carlo error count. The Monte Carlo error count and importance sampling match each other until the error probability of about $2 \times 10^{-5}$, mostly limited by computation resources. Figure 2 shows two simulated points for the case of $\sigma_{t}=1$ using raw Monte Carlo count. Each Monte Carlo simulation counts at least 40 errors to ensure a narrow confident interval. ${ }^{24}$ Figure 2 shows that the method of importance sampling is accurate, at least up to the limitation of the raw Monte Carlo count.

Figure 3 shows the SNR penalty as a function of a normalized timing jitter of $\sigma_{t}$. For lengthening the decision

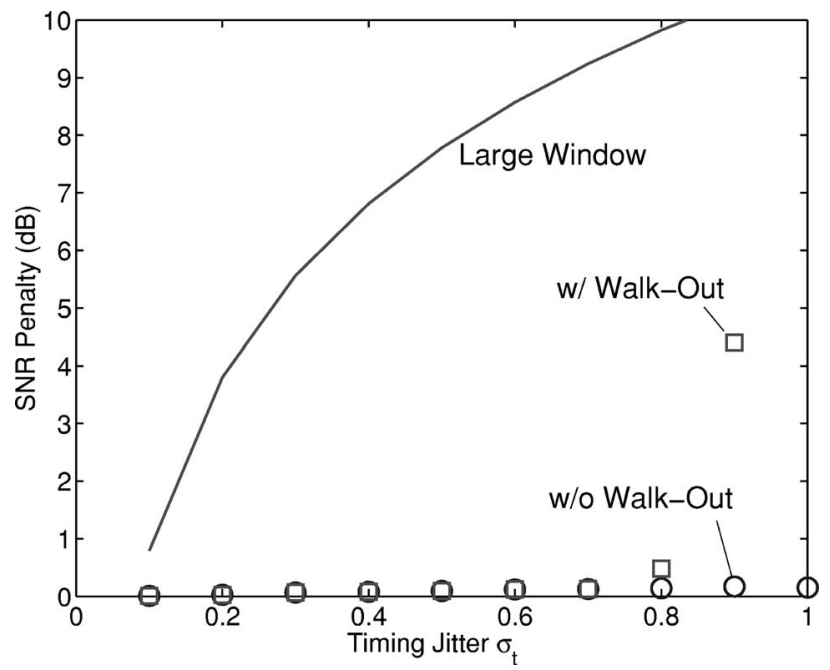

Fig. 3. SNR penalty as a function of a normalized timing-jitter variance of $\sigma_{t}$. The solid curve is using a widening decision window. The square and circle markers are from simulation with and without including the walk-out probability, respectively.

window, the window width must be about $12 \sigma_{t}$ to ensure that the probability of the soliton's walking-out of the decision window is less than $\operatorname{erfc}\left[\tau_{w} /\left(2 \sigma_{t} \sqrt{2}\right)\right]=2 \times 10^{-9}{ }^{1}$. The decision window of $12 \sigma_{t}$ gives $10 \times \log _{10}\left(12 \sigma_{t}\right)$ of SNR penalty when $\sigma_{t}>0.1$. For example, $\sigma_{t}=0.5$ requires a decision window width of $\tau_{w} \approx 6$, corresponding to approximately $7 \mathrm{~dB}$ of SNR penalty. Figure 3 shows that the optimal detector is able to greatly reduce the SNR penalty. For the standard deviation of $\sigma_{t}>1$, the system is dominated by the walk-out probability. With the MLD, walk-out probability is important when $\sigma_{t}>0.8$. For lengthening the decision window, walk-out probability is significant when $\sigma_{t}>0.1$.

The MLD of expression (8) or Fig. 1 has an electrical filter with an impulse response the same as the probability density of $p_{T}\left(t_{0}\right)$. The walk-out probability depends on the tail of $p_{T}\left(t_{0}\right)$, but the left-hand side of expression (8) depends on the center part of $p_{T}\left(t_{0}\right)$ around its mean of $t_{0}=0$. Although the MLD of expression (8) depends weakly on the timing-jitter variance of $\sigma_{t}^{2}$, the walk-out probability depends strongly on $\sigma_{t}^{2}$ as from Fig. 2 .

MLD is an operation in the receiver. Soliton timing jitter is not reduced by using MLD, but the error probability is reduced owing to better soliton detection. The MLD is more applicable when soliton control is used to allow longdistance transmission. Passing through more optical amplifiers than systems without soliton control, the SNR for long-distance systems is reduced. With reduced SNR, the receiver does not allow a large SNR penalty.

\section{CONCLUSION}

The MLD of a soliton with timing jitter is derived, to our knowledge, for the first time. The MLD uses an optical matched filter preceding the photodetector and followed by nonlinear signal processing. The MLD is a process at the receiver that detects the presence or absence of a soliton to minimize the error probability.

With MLD, other than the walk-out probability that the soliton has a timing jitter outside the bit interval, a 
soliton is not affected by timing jitter. Even with a timingjitter standard deviation the same as the soliton FWHM, the SNR penalty is just about $0.2 \mathrm{~dB}$. The MLD has a significantly smaller SNR penalty than a detector with a widening decision window.

K.-P. Ho, the corresponding author, can be reached by e-mail at kpho@cc.ee.ntu.edu.tw.

\section{REFERENCES}

1. J. P. Gordon and H. A. Haus, "Random walk of coherently amplified solitons in optical fiber transmission," Opt. Lett. 11, 865-867 (1986).

2. A. Mecozzi, J. D. Moores, H. A. Huas, and Y. Lai, "Soliton transmission control," Opt. Lett. 16, 1841-1843 (1991).

3. Y. Kodama and A. Hasegawa, "Generation of asymptotically stable optical solitons and suppression of Gordon-Haus effect," Opt. Lett. 17, 31-33 (1992).

4. M. Nakazawa, Y. Yamada, H. Kubota, and E. Suzuki, "10 Gbit/s soliton data transmission over one million of kilometers," Electron. Lett. 27, 1270-1272 (1991).

5. E. Iannone, F. Matera, A. Mecozzi, and M. Settembre, Nonlinear Optical Communication Networks (Wiley, 1998), chap. 5.

6. L. F. Mollenauer, E. Lichtman, M. J. Neubelt, and G. T. Harvery, "Demonstration, using sliding-frequency guiding filters, of error-free soliton transmission over more than 20 $\mathrm{Mm}$ at $10 \mathrm{Gbit} / \mathrm{s}$ single channel, and over more than 13 $\mathrm{Mm}$ at $20 \mathrm{Gbit} / \mathrm{s}$ in a two-channel WDM," Electron. Lett. 29, 910-912 (1993).

7. M. Suzuki, H. Tanaka, N. Edagawa, and Y. Matsushima, "New applications of a sinusoidally driven InGaAsP electroabsorption modulator to in-line optical gates with ASE noise reduction effect," J. Lightwave Technol. 10, 1912-1918 (1992).

8. L. F. Mollenauer, P. V. Mamyshev, and M. J. Neubelt, "Demonstration of soliton WDM transmission at 6 and 7 $\times 10 \mathrm{Gbit} / \mathrm{s}$, error free over transoceanic distance," Electron. Lett. 32, 471-472 (1996).

9. R. N. McDonough and A. D. Whalen, Detection of Signals in Noise, 2nd ed. (Academic, 1995).

10. W. A. Atia and R. S. Bondurant, "Demonstration of returnto-zero signaling in both OOK and DPSK formats to improve receiver sensitivity in an optically preamplifier receiver," in Proceedings of IEEE Laser Electro-Optics Annual Meeting (LEOS) '99 (IEEE, 1999), paper TuM3.
11. D. O. Caplan and W. A. Atia, "A quantum-limited opticallymatched communication link," in Optical Fiber Communication Conference, Vol. 56 of OSA Trends in Optics and Photonics Series (Optical Society of America, 2001), paper MM2.

12. A. Mecozzi, C. B. Clausen, and M. Shtaif, "Analysis of intrachannel nonlinear effects in highly dispersed optical pulse transmission," IEEE Photon. Technol. Lett. 12, 292-294 (2000).

13. M. J. Ablowitz and T. Hirooka, "Intrachannel pulse interaction in dispersion-managed transmission systems: timing shifts," Opt. Lett. 26, 1846-1848 (2001).

14. O. V. Sinkin, V. S. Grigoryan, R. Holzlohner, A. Kalra, J. Zweek, and C. R. Menyuk, "Calculation of error probability in WDM RZ systems in presence of bit-pattern-dependent nonlinearity and of noise," in Optical Fiber Communication Conference, Vol. 95 of OSA Trends in Optics and Photonics Series (Optical Society of America, 2004), paper TuN4.

15. Y. S. Kivshar and B. A. Malomed, "Dynamics of solitons in nearly integrable systems," Rev. Mod. Phys. 61, 763-915 (1989).

16. D. J. Kaup, "Perturbation theory for solitons in optical fibers," Phys. Rev. A 42, 5689-5694 (1990).

17. T. Georges, "Perturbation theory for the assessment of soliton transmission control," Opt. Fiber Technol. 1, 97-116 (1995).

18. K.-P. Ho, "Non-Gaussian statistics of the soliton timing jitter due to amplifier noise," Opt. Lett. 28, 2165-2167 (2003).

19. R. O. Moore, G. Biondini, and W. L. Kath, "Importance sampling for noise-induced amplitude and timing jitter in soliton transmission systems," Opt. Lett. 28, 105-107 (2003).

20. C. R. Menyuk, "Non-Gaussian correction to the Gordon-Haus distribution resulting from soliton interactions," Opt. Lett. 20, 285-287 (1995).

21. J. I. Marcum, "A statistical theory of target detection by pulsed radar," IRE Trans. Inf. Theory IT-6, 56-267 (1960).

22. Y. Yamamoto, "Receiver performance evaluation of various digital optical modulation-demodulation systems in 0.5 $-10 \mu \mathrm{m}$-wavelength region,” IEEE J. Quantum Electron. QE-16, 1251-1259 (1980).

23. K. S. Shanmugan and P. Balaban, "A modified Monte Carlo simulation technique for the evaluation of error rate in digital communication systems," IEEE Trans. Commun. COM-28, 1916-1924 (1980).

24. M. C. Jeruchim, P. Balaban, and K. S. Shanmugan, Simulation of Communication Systems: Modeling, Methodology, and Techniques, 2nd ed. (Plenum, 2001). 